\title{
Estimación de los beneficios de la gestión activa de la demanda. Revisión del estado del arte y propuestas*
}

\author{
Adela Conchado \\ Pedro Linares \\ Instituto de Investigación Tecnológica, \\ Universidad Pontificia Comillas
}

\begin{abstract}
Resumen
Los programas de Gestión Activa de la Demanda (GAD), al trasladar al consumidor los precios variables de generación, transporte y distribución de electricidad, permiten una mayor eficiencia asignativa en los sistemas de energía eléctrica. Sin embargo, también suponen costes no despreciables si requieren la instalación de contadores inteligentes o sistemas avanzados de comunicación y control. La realización de un análisis coste-beneficio es por tanto fundamental para valorar su conveniencia, aunque esto es complejo por la dificultad para predecir los cambios en la demanda y para evaluar los efectos de tales cambios en el sistema eléctrico. Este artículo presenta una revisión del estado del arte de la cuantificación de beneficios de la GAD y ofrece algunas recomendaciones sobre futuras líneas de investigación.
\end{abstract}

Palabras Clave: Sistema eléctrico, gestión activa de la demanda, análisis coste-beneficio.

Códigos JEL: D12, Q41.

\begin{abstract}
Demand Response (DR) programs, by allowing consumers to receive the variable price signals for the generation, transmission and distribution of electricity, increase the allocative efficiency in power systems. However, they may also entail non-negligible costs, if associated to the installation of smart meters or advanced communication and control systems. A cost-benefit analysis is therefore required to assess whether they should be implemented or not, although this is a complex task, due to the difficulty in predicting changes in demand and in assessing their impacts on the power system. This paper presents a survey of the state of the art of the quantification of DR program benefits, and offers some recommendations on future research.
\end{abstract}

Keywords: Power systems, demand response, cost-benefit analysis.

JEL Classification: D12, Q41.

* Esta investigación ha sido financiada por el proyecto GAD. El proyecto GAD (Gestión Activa de la Demanda), financiado por el Centro para el Desarrollo Tecnológico Industrial del Ministerio de Industria, Turismo y Comercio, tiene como objetivo la investigación y el desarrollo de soluciones para la optimización del consumo eléctrico en consumidores de baja y media tensión. El promotor del proyecto, el Consorcio Estratégico Nacional para la Gestión Activa de la Demanda Eléctrica, liderado por Iberdrola Distribución Eléctrica S. A., está formado por otras 14 empresas: Red Eléctrica de España, Unión Fenosa Distribución, Unión Fenosa Metra, Iberdrola, Orbis Tecnología Eléctrica, ZIV Media, DIMAT, Siemens, Fagor Electrodomésticos, BSH Electrodomésticos España, Ericsson España, GTD Sistemas de Información, Acceda Mundo Digital y Airzone. En el proyecto colaboran además 14 organismos investigadores. 


\section{Introducción}

En el contexto energético actual, caracterizado por una creciente preocupación por la sostenibilidad medioambiental y la seguridad de suministro, la búsqueda de soluciones desde el lado de la demanda cobra cada vez más fuerza. Las medidas de gestión de la demanda, encaminadas a promover tanto la adquisición de equipos de bajo consumo como un uso más eficiente de los mismos, podrían ser piezas clave en el futuro de la energía (IEA, 2008; EC, 2005). En el caso de la electricidad, dado que el coste y el impacto del consumo varían en el tiempo, consumir de forma más eficiente supone no sólo reducir el consumo, sino también gestionar el consumo en el tiempo -a nivel horario al menos.

Evidentemente, para que las medidas de gestión de demanda supongan una mayor eficiencia económica, y no sólo energética, debe verificarse que la situación de partida presente fallos de mercado que impidan una asignación eficiente. Este es desgraciadamente el caso habitual de los mercados energéticos (ver p.ej. Labandeira y Linares, 2010), y también el del caso comentado: en la gran mayoría de los sistemas eléctricos ${ }^{1}$ los consumidores no reciben las señales adecuadas para la gestión temporal de su consumo. La razón fundamental es la inexistencia hasta el momento de tecnologías que permitan, por una parte, trasladar a los consumidores estas señales, y por otra parte, medir su consumo temporal. Esta asimetría en la información constituye un fallo de mercado, por cuanto las decisiones de consumo no recogen de forma adecuada el coste de producción del bien, en este caso la electricidad, en los distintos períodos horarios.

La solución de este fallo de mercado es el objetivo fundamental de los programas conocidos como Gestión Activa de la Demanda (GAD) ${ }^{2}$. Existen muchos tipos de programas de GAD, pero en esencia todos ellos consisten en enviar señales de precio -o de cantidad, que pueden ser equivalentes en función de las circunstancias (Weitzman, 1974)- moduladas temporalmente a los consumidores, para que estos puedan responder desplazando sus cargas eléctricas. Esto ocasionaría, en la mayoría de los casos, una disminución de la demanda en horas punta contrarrestada por un aumento de la demanda en horas valle, con el consiguiente aplanamiento de la curva de demanda. Este aplanamiento a su vez llevaría aparejado un aumento de la eficiencia del sistema de generación eléctrica, y también de los sistemas de transporte y distribución de electricidad.

Aunque la GAD no es un concepto nuevo -de hecho, sería el mecanismo natural en un mercado perfecto-, ha cobrado un renovado interés recientemente en vista de la evolución de los sistemas eléctricos (cada vez más congestionados, con redes más

${ }^{1}$ A lo largo del artículo se utiliza el concepto de sistema eléctrico para referirse conjuntamente a la generación, transporte y distribución de electricidad; mientras que en ocasiones se utiliza el de mercado para referirse a los mercados de generación, cuando el sistema eléctrico está liberalizado como sucede en España.

${ }^{2}$ En inglés, suele emplearse el término Demand Response (DR). 
"inteligentes", y con mayor penetración de generación renovable) y gracias al desarrollo de tecnologías de comunicación y control automático cada vez más avanzadas y menos costosas. En este sentido, aunque la mayoría de programas de GAD implementados hasta la fecha se han limitado a contratos de interrumpibilidad para grandes consumidores, hoy en día es posible pensar en formas más sofisticadas de GAD, incluso a nivel doméstico, donde la instalación de contadores inteligentes podría permitir a los consumidores adaptar su consumo a discreción en respuesta a señales de precio variables en el tiempo y por tanto aumentar la eficiencia asignativa en el sistema eléctrico.

El interés actual en la GAD se traduce en numerosos proyectos de investigación ${ }^{3}$, experiencias piloto y otras iniciativas ${ }^{4}$. En algunas regiones se han realizado estudios para evaluar el potencial o la rentabilidad de las medidas de GAD ${ }^{5}$. Además, muchos países han comenzado a instalar contadores inteligentes o han establecido plazos para su progresiva instalación ${ }^{6}$ (Haney et al., 2009), lo que abrirá el abanico de posibles medidas de GAD y facilitará implantarse.

Sin embargo, y como toda regulación encaminada a la corrección de fallos de mercado, parece necesario someterla a un análisis coste-beneficio, de forma que los costes de la regulación no sean mayores que las ganancias de eficiencia logradas. Así, aunque los programas de GAD pueden suponer mejoras en el bienestar (US DOE, 2006), al reducirse por ejemplo los costes de generación o las necesidades de expansión de las redes, también pueden requerir importantes inversiones, especialmente si se pretende dotar al sistema de una infraestructura de contadores, comunicación y control que facilite la respuesta automática de la demanda a las señales de precio. Sin embargo, la estimación de los beneficios a priori no es una cuestión trivial: es difícil estimar los cambios que se producirán en la demanda, y evaluar los efectos que tales cambios de la demanda originarían en el complejo sistema eléctrico requiere un cuidadoso análisis.

El objetivo de este artículo es presentar una revisión de los trabajos realizados en este campo, mostrando el estado del arte del análisis y cuantificación de los beneficios asociados a los programas de gestión activa de la demanda, todo ello bajo el pa-

${ }^{3}$ Algunos proyectos de investigación relacionados con la GAD: GAD (www.proyectogad.es) en España, Smart-A ( www.smart-a.org) y ADDRESS (www.addressfp7.org) en Europa, Demand Response Research Center (http://drrc.lbl.gov) en los Estados Unidos y IEA Demand Side Management Programme (www.ieadsm.org) a nivel internacional.

${ }^{4}$ RRI (2008) refleja el estado acutal de la GAD en los Estados Unidos. Goldfine et al. (2008) exponen los principales avances en programas e iniciativas de GAD. Faruqui y Sergici (2009) presentan una recopilación de los resultados de las 15 experiencias más recientes con tarifas dinámicas a nivel doméstico.

${ }_{5}^{5}$ Por ejemplo, (FERC, 2006) para los Estados Unidos, (NERA, 2008) para Australia, (Vasconcelos, 2008) para la Unión Europea, y (Navigant, 2005) para Ontario (Canadá).

${ }^{6}$ En Europa, la tasa de penetración de contadores inteligentes supera el $85 \%$ en Italia y el $25 \%$ en Francia. Reino Unido, España, Irlanda, Holanda, Noruega y Francia han establecido planes de instalación con el objetivo de alcanzar cerca del 100\% de penetración de contadores inteligentes en el año 2020 (Faruqui et al., 2009). 
radigma del análisis coste-beneficio habitual en la evaluación de políticas públicas. Tras contextualizar las posibles modalidades de GAD (Sección 2), se identificarán y describirán sus beneficios potenciales (Sección 3) y se revisarán las metodologías para su cuantificación propuestas en la literatura (Sección 4), para finalizar con algunas conclusiones sobre el estado del arte en este campo (Sección 5). A lo largo del artículo, se prestará especial atención a proporcionar referencias útiles sobre los diversos aspectos tratados.

\section{Tipología de programas de GAD}

El envío de las señales correctas de precio a los consumidores puede hacerse de muy distintas formas, lo que da origen a una tipología variada de programas de GAD, que pueden clasificarse atendiendo a diversas consideraciones. La Tabla 1 resume algunas de las clasificaciones propuestas en la literatura utilizando la nomenclatura original en inglés.

Como puede observarse en esta tabla, las medidas de GAD pueden tener objetivos económicos (reducir costes) o de mejora de la fiabilidad del sistema-que en el fondo también supone un objetivo económico, aunque generalmente medido en forma "técnica" (RMI, 2006). La señal enviada al consumidor puede requerir una actuación obligatoria, en el caso de emergencias técnicas del sistema (lo que equivaldría a enviar un precio infinitamente alto), o bien no requerir respuesta

\section{TABLA 1}

TIPOLOGÍA DE PROGRAMAS DE GAD

\begin{tabular}{|r|r|l|l|}
\hline Criterio de clasificación & \multicolumn{2}{|c|}{ Opciones } & Referencias \\
\hline Objetivo & Reliability & Economics & (RMI, 2006) \\
\hline Señal & Emergency-based & Price-based & (Faruqui y Hledik, 2007) \\
\hline Origen de la señal & System-led & Market-led & (IEA, 2003) \\
\hline Tipo de señal & Load response & Price response & (RMI, 2006) \\
\hline Tipo de incentivo & Incentive-based & Time-based rates & (FERC, 2006; \\
& US DOE, 2006) \\
\hline Control & Direct load control & Passive load control & (DTE Energy, 2007) \\
\hline
\end{tabular}

Fuente: elaboración propia. 
-en cuyo caso se usa directamente la señal de precio temporal de la energía (Faruqui y Hledik, 2007). Con un significado similar, la señal que promueve la respuesta puede originarse desde el gestor técnico del sistema eléctrico o desde el mercado de generación (IEA, 2003). Puede distinguirse además entre programas de respuesta a cantidad o a precio (RMI, 2006), según se solicite una determinada reducción de carga o se exponga al consumidor a precios variables de la electricidad (RMI, 2006). Para incentivar la respuesta de la demanda, pueden ofrecerse compensaciones a los consumidores que adapten su consumo de la forma requerida o exponer a los consumidores a tarifas que reflejen la variabilidad del precio de la electricidad en el tiempo (FERC, 2006; US DOE, 2006). Por último, hay programas de control directo de la carga, en los que un operador del sistema controla las reducciones de consumo de forma remota (actuando como un planificador), y programas de control pasivo de la carga, en los que son los propios consumidores (o un dispositivo de gestión automática controlado por ellos) los que deciden las reducciones de carga de forma descentralizada (DTE Energy, 2007).

Simplificando, todo el abanico de programas de GAD puede reducirse a dos tipos, que corresponden a cada una de las columnas de la Tabla 1. Por una parte, las medidas de GAD que buscan mejorar la fiabilidad del sistema suelen requerir reducciones puntuales de carga para hacer frente a emergencias. Por ello, suele tratarse de programas de control directo de la carga gestionados por un operador del sistema en los que se ofrece a los consumidores compensaciones por permitir limitaciones puntuales de su consumo en caso de contingencias. Este es el caso actual de los programas de interrumpibilidad a los que pueden acogerse los clientes de alta tensión ${ }^{7}$. Por otra parte, los programas de GAD que buscan reducir los costes del sistema a medio y largo plazo suelen implantarse por medio de tarifas variables en el tiempo que reflejan el precio de mercado, y son los propios consumidores los que voluntariamente adaptan su consumo en función del precio observado (Haney et al., 2009). En este último grupo de medidas se encuentran las tarifas por bloques (o TOU, del inglés time-of-use), las de precio con periodos críticos (o CPP, del inglés critical-peak pricing) o las de precio en tiempo real (o RTP, del inglés real-time pricing).

Otros factores que afectan a las características de los programas de GAD, recogidos de forma resumida en la Tabla 2, son los siguientes:

- El diseño de un programa GAD dependerá del carácter verticalmente integrado o no del sistema eléctrico, fundamentalmente por la elección de los agentes que enviarán las señales y su coordinación (Borenstein et al., 2002; IEA, 2003).

- De forma similar, en un entorno liberalizado, es importante tener en cuenta si la GAD (o la instalación de tecnologías facilitadoras) está auspiciada por el

${ }^{7}$ En estos casos se considera que los beneficios de la actuación centralizada superan a la habitualmente mayor eficiencia de una gestión descentralizada vía precios. 
regulador o se deja a la iniciativa de los agentes de mercado (NERA, 2008), con los posibles problemas habituales de free-riding.

- El segmento de consumidores que participan en el programa (consumidores industriales, comerciales o domésticos, o subgrupos que pueda haber dentro de estos) es otro factor relevante.

- Por último, la tecnología disponible determina de forma crítica las opciones factibles de GAD. Por ejemplo, los programas de control directo de la carga requieren dispositivos de control remoto, y las tarifas de precio en tiempo real requieren una infraestructura avanzada de contadores inteligentes.

TABLA 2

\section{OTROS FACTORES DIFERENCIADORES EN LOS PROGRAMAS DE GAD}

\begin{tabular}{|r|r|l|}
\hline \multicolumn{1}{|c|}{ Otros criterios } & \multicolumn{2}{|c|}{ Opciones } \\
\hline Estructura del sistema & $\begin{array}{r}\text { Regulado } \\
\text { (verticalmente integrado) }\end{array}$ & $\begin{array}{l}\text { Mercado de generación } \\
\text { y comercialización liberalizado }\end{array}$ \\
\hline $\begin{array}{r}\text { Iniciativa a cargo de } \\
\text { de consumidores }\end{array}$ & $\begin{array}{r}\text { Alta tensión } \\
\text { (industrias y grandes comercios) }\end{array}$ & $\begin{array}{l}\text { Baja tensión (pequeños comercios } \\
\text { y consumidores domésticos) }\end{array}$ \\
\hline $\begin{array}{r}\text { Automatización } \\
\text { de la repuesta }\end{array}$ & $\begin{array}{r}\text { Respuesta manual } \\
\text { (sin tecnologías facilitadoras) }\end{array}$ & $\begin{array}{l}\text { Respuesta automática (contadores } \\
\text { y otros dispositivos “inteligentes") }\end{array}$ \\
\hline
\end{tabular}

Fuente: elaboración propia.

\section{Beneficios potenciales de la GAD}

La gestión activa de la demanda puede suponer ganancias de eficiencia en los mercados de generación, en los sistemas de transporte y distribución, y en las actividades de comercialización. Los beneficios que se materializarán en la práctica dependerán del propósito, diseño y funcionamiento del programa de GAD implementado, así como de otros factores como la estructura del sistema en el que se enmarca y las tecnologías facilitadoras disponibles.

Estas ganancias de eficiencia pueden producirse en la operación del sistema eléctrico, en su expansión, y en el funcionamiento del mercado de generación. En esta sección, se identificarán y describirán los beneficios que pueden alcanzarse con medidas de GAD en cada uno de los tres aspectos de los sistemas eléctricos mencionados: operación, expansión y funcionamiento del mercado de generación. La sección concluirá con algunas consideraciones adicionales sobre la distribución de los bene- 
ficios de la GAD entre los agentes implicados y sobre las tecnologías facilitadoras (en particular, sobre los contadores inteligentes).

\subsection{Operación del sistema}

Los programas de GAD en los que los consumidores responden a señales de precio que reflejan los costes horarios de operación (incluyendo generación y/o transporte y distribución) pueden suponer ganancias de eficiencia en la operación del sistema. Así, se evitará o desplazará parte de la demanda en periodos de elevado coste de generación, con el consiguiente beneficio social. Además, si el coste del impacto ambiental está convenientemente internalizado en el precio de la energía -como sucede al menos parcialmente en la actualidad con el precio del permiso de emisión de $\mathrm{CO}_{2}-$, la respuesta de la demanda atenderá también a razones medioambientales (Spees y Lave, 2007). En cualquier caso, es conveniente señalar que la variación del nivel de emisiones dependerá en gran medida del mix de generación. En sistemas en los que la generación marginal en horas punta es menos contaminante que la generación marginal en horas valle (por ejemplo, gas en punta y carbón en valle, como ocurre en muchos sistemas), el efecto de desplazar parte de la carga de horas punta a horas valle podría suponer un aumento de las emisiones, al menos en el corto plazo (Holland y Mansur, 2007). Sin embargo, si se tienen en cuenta, además de los desplazamientos, los efectos de conservación de la carga (ya que es probable que parte de la carga evitada en periodos de precios elevados no se recupere en otros periodos), cabría esperar que las emisiones, en conjunto, se reduzcan (Conchado y Linares, 2009b).

Otro efecto positivo de la GAD en la operación de los sistemas de generación es su posible contribución a abaratar el balance en tiempo real de producción y demanda, lo cual resulta de especial relevancia en sistemas con elevadas cuotas de generación intermitente (Zibelman y Krapels, 2008). De hecho, se considera que la GAD es una herramienta con gran potencial para mitigar los problemas operacionales asociados a la variabilidad e incertidumbre de la generación renovable (Kärkkäinen y Ikäheimo, 2009).

Este aspecto de la GAD como facilitadora del balance en tiempo real de generación-demanda (no sólo para compensar la intermitencia de la generación renovable sino también el posible fallo de un generador), podría suponer una reducción de las necesidades de reservas rodantes para un determinado nivel de fiabilidad de suministro a corto plazo (o un aumento de la fiabilidad de suministro a corto plazo para un determinado nivel de reservas) (Earle et al., 2009).

En cuanto a la operación de las redes, si se expone a los consumidores a las señales temporales adecuadas, la demanda puede responder para aliviar congestiones en las redes o para evitar apagones en caso de contingencias (Affonso et al., 2006). Además, la GAD puede contribuir a reducir las pérdidas en las líneas (Shaw et al., 2009). Los programas de GAD podrían incluso proporcionar servicios complementarios en la operación de las redes, facilitando el control de tensiones, el ba- 
lance de reactiva, la regulación de frecuencia o la corrección del factor de potencia (Crossley, 2008). Todos estos efectos positivos en las redes podrían suponer, en definitiva, una mejora de la fiabilidad de las redes y de la calidad de suministro, o visto de otra forma, una reducción del coste de alcanzar unos determinados niveles de las mismas ${ }^{8}$.

\subsection{Expansión del sistema}

Como ya se ha mencionado, cabe esperar que las medidas de GAD consigan reducir las puntas de demanda, tanto a nivel local como a nivel global.

A nivel local, dado que las redes se dimensionan para la máxima demanda prevista, el aplanamiento del perfil de demanda podría conllevar una disminución de las necesidades de inversión en refuerzos de redes para un determinado nivel de fiabilidad (o un aumento en la fiabilidad de las redes a largo plazo para un determinado nivel de inversión).

A nivel global, el aplanamiento de la demanda podría reducir las necesidades de inversión en nuevas unidades de generación de punta y de reserva de capacidad (Braithwait et al., 2006) para un determinado nivel de fiabilidad de suministro (o un aumento de la fiabilidad de suministro para un determinado nivel de reservas).

Otro efecto potencial de la GAD que afecta a la expansión de los sistemas de generación es que podría permitir mayores niveles de penetración de generación intermitente, lo cual puede ser considerado un beneficio en países en los que se promociona la generación renovable.

\subsection{Funcionamiento del mercado de generación}

Muchos mercados eléctricos presentan características de oligopolio, por la presencia de un reducido número de productores de electricidad. En estos casos, aumentar la elasticidad de la demanda -al aumentar la respuesta de los consumidores- disminuiría la capacidad de los generadores para ejercer poder de mercado (IEA, 2003; Braithwait et al., 2006), lo que supondría a su vez un disminución en el mark-up de los precios (Kirschen, 2003; Borenstein et al., 2002) y por tanto en la eficiencia del mercado.

Además, también en los mercados en competencia perfecta los precios se moderarían por efecto del aplanamiento de la curva de demanda9 (IEA, 2003). Sin em-

${ }^{8}$ Habitualmente los costes de fiabilidad no son trasladados al consumidor de forma directa, por lo que es difícil asumir que la situación actual ya resulta en una decisión eficiente acerca del nivel de fiabilidad óptimo.

${ }^{9}$ Debido fundamentalmente a la pendiente creciente de las curvas de costes. El aplanamiento de la curva supone una subida de los precios del valle y una reducción de los precios de la punta. Pero la pendiente creciente hace que el efecto global sea habitualmente de reducción en el coste total. 
bargo, debe tenerse en cuenta que la disminución de los precios en este caso tan sólo representa una transferencia de beneficios de los generadores a los consumidores, y no ahorros sociales reales (Braithwait et al., 2006).

Por otra parte, la GAD podría permitir a los generadores y comercializadores reducir los costes de los desvíos (IEA, 2003). De forma similar, la GAD puede verse como una forma de cobertura de los riesgos asociados a la volatilidad de los precios y los niveles de producción (PLMA, 2002) o a situaciones extremas del sistema difícilmente predecibles (Violette et al., 2006a).

Con la implantación de programas de GAD, los comercializadores podrían aumentar sus oportunidades de negocio y ofrecer contratos a los consumidores más ajustados a sus patrones de demanda. Al mismo tiempo, los consumidores se verían beneficiados por una mayor capacidad de elección y podrían ahorrar dinero en su factura eléctrica si su patrón de consumo es favorable al sistema (en el sentido de que su demanda es baja en periodos de elevado coste).

En resumen, la GAD en un contexto de mercado podría suponer mejoras significativas en el funcionamiento del mismo, con posibles ganancias de eficiencia asociadas.

\subsection{Resumen de beneficios}

A continuación, la Tabla 3 resume los beneficios potenciales de la GAD que han sido mencionados a lo largo de la sección, categorizados en función de la actividad de los sistemas eléctricos en la que se originan. Obsérvese que los beneficios se asignan a la actividad en la que se originan sin considerar la actividad que finalmente los percibiría (la distribución de los beneficios entre los agentes se discutirá en 3.5). En relación con esto, los beneficios incluidos en la Tabla 3 son sólo aquellos que representan ahorros reales o mejoras de eficiencia para la sociedad en su conjunto, y no transferencias de beneficios entre los agentes.

TABLA 3

RESUMEN DE BENEFICIOS POTENCIALES DE LA GAD

\begin{tabular}{|l|l|l|l|}
\hline & \multicolumn{1}{|c|}{ Operación } & \multicolumn{1}{c|}{ Expansión } & Mercado* $^{*}$ \\
\hline & $\begin{array}{l}\text { - Alivio de congestiones } \\
\text { - Gestión } \\
\text { de contingencias, }\end{array}$ & $\begin{array}{l}\text { - Retraso de inversiones } \\
\text { en refuerzos de redes } \\
\text { o aumento de la } \\
\text { Transmisión }\end{array}$ & \\
y distribución & evitar apagones & & \\
& - Reducción de las & a largo plazo la red & \\
& pérdidas & & \\
& Contribución a la & & \\
& operación técnica & & \\
\hline
\end{tabular}


TABLA 3 (continuación)

RESUMEN DE BENEFICIOS POTENCIALES DE LA GAD

\begin{tabular}{|c|c|c|c|}
\hline & Operación & Expansión & Mercado* \\
\hline Generación & $\begin{array}{l}\text { - Reducción de la } \\
\text { generación en horas } \\
\text { punta: reducción } \\
\text { del coste de la energía } \\
\text { y (posiblemente) } \\
\text { de las emisiones }{ }^{\text {b }} \\
\text { - Contribución } \\
\text { al balance } \\
\text { producción-demanda } \\
\text { (especial relevancia } \\
\text { con generación } \\
\text { intermitente) } \\
\text { - Disminución de la } \\
\text { necesidad de reserva } \\
\text { rodante o aumento de } \\
\text { la fiabilidad a corto } \\
\text { plazo }\end{array}$ & $\begin{array}{l}\text { - Ahorro en inversión } \\
\text { en unidades de } \\
\text { generación de punta } \\
\text { - Ahorro en reservas de } \\
\text { capacidad o aumento } \\
\text { de la fiabilidad } \\
\text { a largo plazo } \\
\text { - Permitir mayor } \\
\text { penetración } \\
\text { de generación } \\
\text { intermitente } \\
\text { (renovables) }^{\mathrm{c}}\end{array}$ & $\begin{array}{l}\text { - Disminución del riesgo } \\
\text { de desvíos } \\
\text { - Mitigación del poder } \\
\text { de mercado } \\
\text { - Disminución } \\
\text { de la volatilidad } \\
\text { de los precios }\end{array}$ \\
\hline Comercialización* & & & $\begin{array}{l}\text { - Disminución del riesgo } \\
\text { de desvíos } \\
\text { - Reducción de la } \\
\text { volatilidad de los } \\
\text { precios } \\
\text { - Nuevos productos, } \\
\text { más capacidad de } \\
\text { elección por parte } \\
\text { de los consumidores }\end{array}$ \\
\hline Demanda & $\begin{array}{l}\text { - Mayor conocimiento } \\
\text { del consumo y los } \\
\text { costes, e incluso de los } \\
\text { impactos ambientales } \\
\text { - Opción para que los } \\
\text { consumidores } \\
\text { maximicen su utilidad: } \\
\text { oportunidad para } \\
\text { reducir su gasto } \\
\text { en electricidad }\end{array}$ & $\begin{array}{l}\text { - Decisiones } \\
\text { de inversión con } \\
\text { mayor conocimiento } \\
\text { de consumo y coste }\end{array}$ & $\begin{array}{l}\text { - Aumento } \\
\text { de la elasticidad } \\
\text { de la demanda }\end{array}$ \\
\hline
\end{tabular}

* Sólo aplicable en entornos liberalizados.

a Mantener niveles de frecuencia y tensión, compensar potencia activa y reactiva, controlar el factor de potencia, etc.

b Depende del mix de generación.

c Puede considerarse un beneficio en sistemas en los que se promociona la generación renovable.

Fuente: elaboración propia. 


\subsection{Consideraciones adicionales}

3.5.1. Distribución de los beneficios entre los agentes

Los beneficios de la GAD originados en los sistemas de generación y redes no serán necesariamente percibidos por las empresas generadoras y los operadores de las redes, respectivamente. La distribución de los beneficios entre los agentes es una cuestión clave a la hora de evaluar los programa de GAD, y necesita ser cuidadosamente analizada considerando el marco regulatorio aplicable.

En términos generales, en un sistema eléctrico no liberalizado, la distribución de los beneficios dependerá de la determinación de las tarifas por parte del regulador.

En sistemas liberalizados, si el esquema de remuneración está basado en incentivos, los beneficios originados en las redes de distribución serían percibidos a corto plazo por las compañías distribuidoras, y transferidos a los consumidores a largo plazo por medio de reducciones en las tarifas de acceso. Por el contrario, los ahorros originados por la GAD en el sistema de generación serían transferidos directamente a los consumidores por medio de precios de la electricidad más bajos (si los mercados son competitivos), suponiendo al mismo tiempo una disminución de los ingresos de las compañías generadoras. En cualquier caso, e incluso suponiendo un mercado oligopolista, un uso más eficiente de la energía y una mayor elasticidad en su demanda deberían traducirse en mejoras del bienestar para los consumidores (ERGEG, 2007).

Según IEA (2003), la distribución de beneficios entre los agentes en entornos liberalizados supone una dispersión de los incentivos para promover o participar en programas de GAD de la siguiente forma:

- Los generadores de base tienen escasos incentivos, podrían estar interesados en medidas de GAD únicamente como forma de cobertura del riesgo de fallos no previstos de sus unidades de generación. En cambio, los generadores de punta perciben la GAD como un foco directo de competencia.

- Los operadores del sistema podrían estar interesados en la GAD para facilitar el balance producción-demanda y para mejorar la fiabilidad del sistema.

- Los operadores de las redes podrían utilizar programas de GAD para aliviar congestiones en sus redes, mejorar la fiabilidad o la calidad de suministro a nivel local, o reducir inversiones en refuerzos, pero sus incentivos dependerán de forma crucial en el marco regulatorio vigente.

- Los comercializadores podrían hacer uso de la GAD para equilibrar el suministro contratado con la demanda de sus clientes.

- Los incentivos de los consumidores dependerían básicamente de los precios o compensaciones ofrecidos por los comercializadores. 


\subsubsection{Contadores inteligentes y otras tecnologías facilitadoras}

La instalación de tecnologías facilitadoras puede ampliar y amplificar los beneficios potenciales de la GAD. De hecho, la mayor parte de los beneficios recogidos en la Tabla 3 sólo pueden materializarse si se dispone de una infraestructura de contadores avanzados y/o control. Así, la implantación de tarifas horarias requiere la existencia de contadores inteligentes y sistemas de comunicación, y las medidas de interrupción de la carga aplicadas para gestionar de contingencias requieren dispositivos de control remoto.

En la literatura, gestión activa de la demanda y smart metering (contadores inteligentes) son conceptos relacionados. Muchos estudios que han evaluado la rentabilidad de los contadores inteligentes tienen en cuenta beneficios asociados a la GAD (por ej.: CapGemini, 2007; Ofgem, 2006; Frontier Economics, 2006; Haney et al., 2009). De hecho, los beneficios de la GAD representan la mayor parte de los beneficios sociales atribuidos a los contadores inteligentes en análisis recientes (Neenan y Hemphill, 2008). Otros estudios que han analizado los contadores inteligentes desde una perspectiva más regulatoria pueden aportar también interesantes indicaciones sobre la GAD (por ej.: EEI, 2006; ERGEG, 2007).

Sin embargo, cabe señalar que la instalación de contadores inteligentes supondría algunos beneficios operacionales no relacionados con la GAD, tales como ahorros en la lectura de los contadores y en la detección de faltas en las redes. Estos ahorros deberían tenerse en cuenta al evaluar la rentabilidad de las infraestructuras de contadores avanzados, pero no se considerarán en la revisión de este artículo.

Haney et al. (2009) mencionan los siguientes beneficios operacionales de los contadores inteligentes:

- Mejora de la eficiencia de los servicios de lectura: costes evitados de lectura de contadores, mejora en la detección de faltas, tiempos de respuesta ante faltas más cortos, mejora de los registros de calidad de suministro y precisión en la facturación.

- Ahorros en los costes de atención a los consumidores.

- Reducción de las pérdidas no técnicas.

- Otros, como aumento de la capacidad de elección de opciones de pago, mejora de la información de consumo o facilitación de la microgeneración.

Además, los contadores inteligentes permitirían disponer de más información a nivel nodal y adecuar las tarifas de uso de las redes para reflejar los costes incurridos (IEA, 2003). La posibilidad de conocer los patrones de demanda podría permitir planificar la operación y expansión de las redes de forma más eficiente (simplemente por el valor de la información, sin considerar GAD). Por último, los contadores inteligentes permitirían la implantación de tarifas innovadoras y más competencia en las actividades de comercialización (Frontier Economics, 2006). 
Además de los contadores inteligentes, existen otras tecnologías que podrían contribuir a gestionar la demanda, tales como termostatos inteligentes ${ }^{10}$, sistemas de control automático de la iluminación, sistemas térmicos de almacenamiento o relés de frecuencia o tensión (Batlle y Rodilla, 2008). Lockheed Martin Aspen (2006) analiza en detalle el estado actual de las tecnologías facilitadoras a nivel residencial o de pequeño comercio, y SCE (2006) presenta un inventario de tecnologías emergentes para la GAD.

\section{Cuantificación de beneficios de la GAD}

Cuantificar los beneficios de la GAD requiere en primer lugar una estimación de los cambios inducidos en la curva de demanda, y en segundo lugar un cuidadoso análisis del efecto de tales cambios en el sistema eléctrico. Las siguientes secciones (4.1 y 4.2) presentan una revisión de estudios previos que han contribuido con sus aportaciones a conocer mejor cada uno de estos dos aspectos.

\subsection{Estimación de cambios en la demanda}

La mayor parte de los beneficios asociados a los programas de GAD dependen directamente de los cambios que pueden inducir en la demanda. Por ello, para cuantificar los beneficios de la GAD es fundamental estimar en primer lugar los efectos de conservación y desplazamiento de cargas que podría suponer en la demanda de los consumidores participantes. En general, es necesario evaluar cada segmento de consumidores por separado para tener en cuenta las diferencias en los patrones de consumo y en las sensibilidades al precio.

Debe tenerse en cuenta además que el nivel de detalle con el que se evalúen los cambios de demanda debe ser coherente con la metodología que se aplicará posteriormente para cuantificar los beneficios. Para algunos estudios sencillos basados en estimaciones, puede ser suficiente evaluar la variación en bloques discretos de demanda (por ejemplo, punta y valle), mientras que análisis más complejos basados en simulaciones frecuentemente requieren curvas de demanda a nivel horario (o incluso cuartohorario).

Es importante además tener en cuenta la dificultad de predecir cambios inciertos en la demanda. La respuesta de los consumidores tiene carácter aleatorio, y puede verse influenciada por numerosos factores. En los estudios realizados hasta la fecha se destacan como posibles factores el clima, el diseño tarifario o el nivel de precios, el tipo de consumidor (equipos eléctricos disponibles, presupuesto, nivel de consu-

${ }^{10}$ El programa Smart Thermostat es una interesante experiencia en California en la que se utilizaron termostatos inteligentes para controlar los equipos de aire acondicionado de 5.000 consumidores domésticos (KEMA-XENERGY, 2003). 
mo, etc.), las tecnologías facilitadoras disponibles, el medio de notificación de los precios o las alertas, la información recibida por los consumidores y las campañas de concienciación que se promuevan (Kohler y Mitchell, 1984; Faruqui y George, 2005; Herter, 2007; EEE, 2006; Summit Blue, 2006; Darby, 2006).

A continuación se presentan tres posibles métodos para evaluar los efectos de la GAD en el perfil de la demanda:

i) emplear estimaciones aportadas por estudios previos (fundamentalmente, elasticidades demanda-precio),

ii) desarrollar un modelo econométrico de la demanda, y

iii) simular la demanda con un modelo tecnológico o bottom-up.

En la literatura pueden encontrarse otras revisiones de métodos para evaluar la respuesta de la demanda, como la de Woo y Herter (2006).

\subsubsection{Estimaciones de la elasticidad demanda-precio}

Una forma directa y relativamente sencilla de evaluar los cambios provocados por medidas de GAD en la demanda consiste en utilizar medidas de la elasticidad demanda-precio estimadas en estudios previos. La elasticidad demanda-precio expresa el incremento de la demanda en términos porcentuales ante un incremento del $1 \%$ en el precio.

En el contexto de la GAD, se utilizan tres tipos de elasticidad: propia, cruzada y de sustitución. La elasticidad propia expresa la variación de la demanda en un periodo ante un incremento del $1 \%$ en el precio de ese mismo periodo, mientras que la elasticidad cruzada expresa la variación de la demanda en un periodo ante un incremento del $1 \%$ en el precio de otro periodo. La elasticidad de sustitución, en cambio, expresa la demanda desplazada de un periodo a otro ante un incremento del $1 \%$ en el precio relativo entre ambos (considerándose habitualmente entre un periodo punta y un periodo valle) (King y Chatterjee, 2003).

Algunos autores han recogido medidas de elasticidad de estudios previos para diferentes tipos de programa de GAD y diferentes regiones, o han presentado sus propias estimaciones. A continuación, la Tabla 4 presenta algunos valores de elasticidades propias y de sustitución (adaptados de US DOE, 2006), en función del segmento de consumidores participantes y el tipo de tarifa aplicado.

Numerosos estudios han empleado este tipo de parámetros de elasticidad para estimar los cambios de la demanda en evaluaciones de medidas de GAD. Por ejemplo, Berg et al. (1983) emplean elasticidades horarias propias y cruzadas (tanto a corto plazo como a largo plazo), mientras que Navigant (2005) utiliza elasticidades de sustitución para calcular los desplazamientos de carga en el perfil de demanda de diversos segmentos de consumidores. 


\section{TABLA 4}

\section{RESUMEN DE ESTIMACIONES DE ELASTICIDAD DEMANDA-PRECIO (ADAPTADO DE US DOE, 2006)}

\begin{tabular}{|c|c|c|c|c|}
\hline $\begin{array}{c}\text { Segmento } \\
\text { de consumidores }\end{array}$ & Tipo de tarifa & Elasticidad propia & $\begin{array}{c}\text { Elasticidad } \\
\text { de sustitución }\end{array}$ & Región \\
\hline \multirow{4}{*}{$\begin{array}{c}\text { Domésticos } \\
\text { (y pequeño comercio) }\end{array}$} & TOU & & $\begin{array}{c}0,07 \text { a } 0,21 \\
\text { (media } 0,14 \text { ) }\end{array}$ & EEUU \\
\hline & TOU / CPP & $\begin{array}{l}-0,1 \text { a }-0,8 \\
\text { (media }-0,3 \text { ) }\end{array}$ & & $\begin{array}{c}\text { EEUU - } \\
\text { Internacional }\end{array}$ \\
\hline & $\mathrm{CPP}$ & & $\begin{array}{l}0,04 \text { to } 0,13 \\
\text { (media } 0,09)\end{array}$ & California \\
\hline & RTP & $\begin{array}{l}-0,05 \text { a }-0,12 \\
(\text { media }-0,08)\end{array}$ & & Illinois \\
\hline \multirow{5}{*}{$\begin{array}{c}\text { Pequeño } \\
\text { y mediano comercio } \\
\text { e industria }\end{array}$} & \multirow{5}{*}{ RTP } & $-0,01$ a $-0,28$ & & Georgia \\
\hline & & $-0,01$ a $-0,27$ & & Reino Unido \\
\hline & & $<-0,01$ a $-0,38$ & & Carolina del N.-S. \\
\hline & & & 0,10 a 0,27 & Sudoeste EEUU \\
\hline & & & $\begin{array}{c}0,02 \text { a } 0,16 \\
\text { (media } 0,11)\end{array}$ & Nueva York \\
\hline
\end{tabular}

\subsubsection{Modelos econométricos}

Los modelos econométricos tratan de estimar el nivel de demanda a partir de algunos factores explicativos basándose en la teoría microeconómica. La formulación habitual de este tipo de modelos es la maximización de la utilidad del consumo eléctrico para los consumidores. Los factores explicativos más frecuentes son el precio de la electricidad y el presupuesto del consumidor. Pueden tenerse en cuenta también condiciones sociodemográficas o aspectos tecnológicos. Los modelos econométricos suelen desarrollarse a partir de datos de experiencias reales, y luego emplearse para evaluar otros programas similares. Además, estos modelos suelen permitir obtener medidas de la elasticidad demanda-precio.

Algunos ejemplos tempranos de este tipo de enfoque son los estudios de Lawrence y Braithwait (1979) y de Hausman et al. (1979), que desarrollan un modelo econométrico para analizar el efecto de las tarifas TOU. En ambos estudios, la demanda viene determinada por la maximización de la utilidad del consumidor sujeta a una 
restricción de presupuesto. La demanda en diferentes periodos se considera como un bien diferente, de tal forma que los desplazamientos de carga pueden modelarse como sustituciones entre dos bienes.

Los estudios de Caves et al. (1984), Parks y Weitzel (1984) y Hill (1991), también para TOU, son similares. Más recientemente, el modelo propuesto por Reiss y White (2005) permite la evaluación de diferentes diseños tarifarios.

Desde una perspectiva diferente, Allcott (2008) estima la demanda horaria residencial con una función de utilidad que depende de las características de la vivienda, el precio de la electricidad a lo largo del día y algunos parámetros de sustitución y desplazamiento de cargas (basados en la temperatura y variables dummy).

A diferencia de los estudios ya mencionados, Medlock (2004) incluye consideraciones técnicas de los equipos para valorar su consumo de energía en la producción de bienes y servicios. El autor propone una metodología general para estimar la energía consumida como una función de los equipos disponibles, de su utilización y de su eficiencia.

Los modelos econométricos pueden proporcionar una representación precisa de la demanda si se incluyen los factores que afectan a la demanda de forma más significativa, y si los parámetros que expresan cómo varía la demanda en función de tales factores se estiman adecuadamente. Sin embargo, el modelo pude dejar de ser válido si cambian las condiciones para las cuales se realizó el ajuste ${ }^{11}$. Así, la principal limitación de este tipo de enfoque es que sus resultados son muy dependientes de las condiciones subyacentes y difíciles de extrapolar.

Los modelos econométricos pueden incluir o no consideraciones tecnológicas. Sin embargo, una representación de la demanda que ignore la heterogeneidad de las cargas eléctricas y sus restricciones técnicas puede no ser suficiente para evaluar medidas de GAD. La utilidad del consumo eléctrico depende de su uso final (el equipo eléctrico utilizado) y de aspectos circunstanciales (el servicio proporcionado por el equipo eléctrico en el momento del consumo). Además, la capacidad de desplazar o reducir las cargas depende muy notablemente del potencial técnico de los equipos eléctricos en uso. Por lo tanto, incluir consideraciones tecnológicas en los modelos econométricos podría mejorar notablemente la representación de la respuesta de la demanda.

\subsubsection{Modelos tecnológicos o bottom-up}

Los modelos tecnológicos o bottom-up, a diferencia de los modelos econométricos, tratan de capturar las cargas específicas que constituyen la demanda. El perfil de demanda se obtiene por agregación de cargas elementales, que pueden representar consumidores individuales o el consumo de cada electrodoméstico.

Hay algunos estudios interesantes que han modelado la demanda eléctrica utilizando este tipo de enfoque pero sin considerar acciones de GAD. Es el caso de Cappasso

${ }^{11}$ En la línea de la crítica de Lucas a los modelos macroeconómicos (Lucas, 1976). 
et al. (1994) y Boonekamp (2007) para la demanda doméstica. Aunque estos modelos no serían aplicables a la evaluación de programas de GAD, sus contribuciones pueden resultar de utilidad para desarrollar modelos adaptados al análisis de la GAD.

Otros estudios han incorporado en sus modelos bottom-up la posibilidad de desplazar o reducir la demanda suponiendo programas de control directo de la carga. El trabajo de Paatero y Lund (2006) es un buen ejemplo. Los autores utilizan datos estadísticos para representar el consumo doméstico, e incorporan procesos estocásticos para tener en cuenta la naturaleza aleatoria de la demanda.

Por último, algunos estudios han considerado la respuesta de la demanda a precios de forma explícita. Conchado y Linares (2009a) propone distinguir en la curva de demanda de una vivienda el consumo de los diferentes electrodomésticos disponibles (utilizando para ello perfiles simplificados de sus ciclos de trabajo), y desplazar o reducir el consumo de cada electrodoméstico en función de su potencial técnico y el precio horario de la electricidad (con el objetivo de minimizar el coste del consumo). Con un enfoque diferente, Lu et al. (2004) modelan las cargas controladas termostáticamente por medio de circuitos eléctricos equivalentes que representan sus propiedades térmicas, y las cargas no controladas termostáticamente como tareas en un sistema de colas.

\subsection{Cuantificación de beneficios}

La cuantificación de beneficios de la GAD ha sido abordada desde diversas perspectivas y metodologías. Por ejemplo, Heffner (2007) distingue seis tipos de estudios: estimación de costes evitados, planificación de recursos, análisis del excedente social, valoración de la fiabilidad del sistema, planificación de las redes de transporte, y subastas de capacidad. La conveniencia de cada método dependerá del tipo de beneficio a evaluar y de su contexto, no existiendo ningún método capaz de recoger con precisión todo el rango de posibles efectos de la GAD en los sistemas eléctricos.

En esta sección se presenta una revisión del estado del arte de la cuantificación de los beneficios de las medidas de GAD, distinguiendo entre dos tipos de estudios: los basados en estimaciones y los basados en simulaciones. Ambos enfoques presentan ventajas e inconvenientes. Los estudios basados en estimaciones son simples y transparentes, pero es probable que no representen con suficiente precisión la complejidad de los sistemas eléctricos. Por el contrario, los análisis basados en simulaciones permiten representar los sistemas eléctricos en detalle, pero sus resultados son menos transparentes y difíciles de comparar.

\subsubsection{Estudios basados en estimaciones}

En este tipo de estudios, los beneficios de la GAD se calculan de forma analítica a partir de algunas estimaciones, realizando necesariamente simplificaciones sobre 
el comportamiento de los sistemas eléctricos que permitan expresar todas las interacciones en términos algebraicos. En general, suelen considerar tan solo unos pocos periodos (tales como punta y valle).

Un ejemplo ilustrativo de este tipo de análisis puede encontrarse en el estudio de Baer et al. (2004), en el que se evalúa la iniciativa GridWise en los EEUU. A partir de un conjunto de datos de entrada (elasticidad demanda-precio por segmentos de consumidores, precios de mercado en punta y valle en un escenario base, margen de reserva de generación previsto en 2025, tasa de descuento, etc.), se estima la magnitud de la reducción de la punta de demanda. Con esta estimación, los ahorros en inversiones de generación se calculan simplemente multiplicando la reducción de la punta de carga por el coste de inversión de las unidades de generación de punta (turbinas de gas o generadores diésel). De forma similar, los ahorros en combustible y operación y mantenimiento se calculan multiplicando la reducción de la punta de carga por los costes respectivos representativos de las centrales evitadas. Los ahorros en inversiones de redes se estiman directamente como una fracción de los ahorros en inversiones de generación.

Otro ejemplo de estudio basado en estimaciones es el análisis coste-beneficio de los contadores inteligentes en Francia realizado por CapGemini (2007). Se consideran tres escenarios representando diferentes niveles tecnológicos, y se supone que el nivel de respuesta de la demanda depende del nivel de prestaciones tecnológicas de los contadores. Las estimaciones finales de los beneficios se asignan a generadores, distribuidores y comercializadores, lo cual resulta muy útil para la regulación del modelo.

Otros ejemplos de este tipo de análisis son los estudios de Faruqui et al. (2009) para la Unión Europea, Ofgem (2006) para el Reino Unido, Siderius y Dikstra (2006) para Holanda, Faruqui y George (2002) para Estados Unidos, Navigant (2005) para Ontario, o ESC (2004) para Australia.

Los puntos fuertes de este enfoque son su simplicidad y transparencia. Las relaciones entre los supuestos realizados y los resultados obtenidos pueden explorarse fácilmente, y resulta fácil comparar los resultados de diversas alternativas de GAD. Su inconveniente, sin embargo, es que puede no representar con precisión las numerosas y complejas interacciones que tienen lugar en los sistemas eléctricos. Además, al considerar tan sólo estimaciones puntuales de los parámetros (y no incorporar en ningún caso consideraciones estocásticas), la dependencia de los resultados de los supuestos de partida se magnifica respecto a otros métodos (Neenan y Hemphill, 2008).

\subsubsection{Estudios basados en simulaciones}

En lugar de utilizar estimaciones, los beneficios de la GAD pueden evaluarse con la ayuda de modelos que simulan el comportamiento de los sistemas eléctricos. Dado que los modelos de simulación permiten una representación detallada de la opera- 
ción y/o expansión de los sistemas de generación y las redes, o del funcionamiento del mercado, este enfoque parece ser el más preciso para la cuantificación de beneficios de la GAD.

En los estudios basados en simulaciones, los beneficios de la GAD se calculan generalmente como la diferencia en los resultados de dos simulaciones, una para un escenario base sin GAD y otra para un escenario con GAD.

Hasta la fecha, la mayoría de los estudios que han evaluado los beneficios de la GAD con la ayuda de modelos de simulación se han centrado en el análisis de los impactos en el sistema de generación o en el funcionamiento del mercado, pero existen también algunos estudios que han abordado el análisis de los impactos en las redes. En esta sección se revisará el estado del arte en ambos campos, presentando en primer lugar la evaluación de los impactos en generación y en segundo lugar la evaluación de los impactos en las redes.

\section{a) Sistema de generación}

En la literatura, pueden encontrarse estudios que han evaluado la GAD tanto en un contexto de sistema regulado (por ej. Berg et al., 1983) como en un contexto de mercado liberalizado, suponiendo competencia perfecta (por ej. Borenstein, 2005) o competencia imperfecta (por ej. Allcott, 2008).

La respuesta de la demanda puede incorporarse de forma endógena en los modelos de simulación, bien sea considerando curvas de demanda elásticas en modelos de equilibrio de mercado (por ej. Su y Kirschen, 2009) o considerada como un recurso disponible en la planificación de un sistema centralizado (por ej. Violette et al., 2006b). En cambio, otros estudios determinan la variación de la demanda de forma exógena, suponiendo ciertas reducciones de carga (por ej. Brattle, 2007) o evaluando ex-ante los cambios en el perfil de carga con la ayuda de modelos (por ej. Linares y Conchado, 2009).

Algunos autores han considerado la aleatoriedad del valor futuro de las variables más relevantes mediante simulaciones estocásticas, como Andersen et al. (2006), quienes utilizan simulaciones de Monte Carlo (siguiendo a Violette et al., 2006b) para evaluar el potencial de las medidas de GAD no sólo en situaciones promedio si no también en situaciones extremas.

Para tener en cuenta las congestiones en las redes, es posible incluir en el modelo de generación una representación de la red de transporte y simular los flujos de potencia por las líneas. Los impactos de la GAD se pueden entonces estimar utilizando los precios nodales marginales, como en el estudio de Neenan et al. (2005) o Brattle (2007). Wallawalkar et al. (2008) también emplea precios nodales marginales en su análisis del excedente social, pero los calcula por medio de un modelo econométrico.

El potencial de la GAD como facilitadora del balance de generación y demanda en tiempo real en sistemas con elevada penetración de generación eólica ha sido 
investigado por Sioshanshi y Short (2009) y Silva (2009). Los primeros autores cuantifican la reducción de los vertidos eólicos suponiendo que la demanda es elástica al precio en tiempo real, y que éste refleja adecuadamente la disponibilidad de generación eólica y posibles restricciones en las redes que afectan a su transporte hasta los puntos de demanda. Silva (2009), en cambio, evalúa la contribución de la GAD al balance generación-demanda considerando la respuesta de la demanda como una reserva despachable que contribuye a minimizar los costes del sistema.

\section{b) Redes de transporte y distribución}

Existen pocos estudios de cuantificación de los impactos de la GAD en las redes de transporte y distribución.

El impacto de la GAD en las necesidades de inversión de las redes de distribución ha sido estimado por Conchado y Linares (2009b) empleando un modelo de simulación de la expansión de las redes de distribución y suponiendo ciertas reducciones en la máxima potencia demandada por los consumidores participantes.

En relación con la operación de las redes, Shaw et al. (2009) han estimado la reducción de las pérdidas en las líneas de distribución debidas al desplazamiento de las cargas domésticas. Para ello, han simulado cómo afectan los cambios en el perfil de demanda a los flujos de potencia en una red de distribución y han supuesto que el potencial de reducción de las pérdidas depende del nivel de pérdidas preexistente y de los patrones de demanda.

El papel de la GAD para aliviar congestiones en las redes ha sido analizado por Stanojević y Silva (2009) y Stanojević et al. (2009). En el primer estudio, se propone utilizar un modelo de flujo óptimo de cargas que incorpora un modelo de respuesta de la demanda (considerando gestión térmica de la carga y desplazamientos de electrodomésticos a nivel residencial) para estimar la reducción de los costes de congestión al modificarse el perfil de demanda. De forma similar, Stanojević et al. (2009) demuestran cómo la variación de la curva de demanda puede mejorar la utilización de la capacidad de distribución existente y reducir los niveles críticos de carga en una red sobrecargada. El modelo empleado para ello combina el despacho de las unidades de generación con un modelo de flujo de cargas multiperiodo donde puede hacerse uso de la respuesta de la demanda para minimizar el redespacho de unidades.

\section{Conclusiones}

Los programas de gestión activa de la demanda, enmarcados generalmente en un contexto de redes inteligentes, muestran un gran potencial y un futuro prometedor, especialmente si se consiguen materializar los beneficios que podrían supo- 
ner para los sistemas de generación, las redes, los comercializadores y los consumidores en términos de ganancia de eficiencia. Sin embargo, también pueden suponer costes no despreciables asociados a la infraestructura de comunicaciones necesaria. Por ello, en la actualidad se están realizando numerosos estudios para valorar sus beneficios potenciales, tanto utilizando estimaciones más o menos simplificadas como enfoques más complejos basados en modelos econométricos o simulaciones. En este artículo se ha presentado una revisión exhaustiva del estado del arte de estos análisis.

Cuantificar los beneficios de medidas de GAD requiere, en primer lugar, estimar los cambios que podrían producirse en la demanda, y en segundo lugar, evaluar el impacto de tales cambios en el sistema eléctrico (generación y redes). Aunque se han identificado numerosos estudios en este campo, y dada la falta de madurez de estos programas, se considera que es necesario seguir investigando para proporcionar estimaciones más fiables de los beneficios de la GAD, y así poder valorar rigurosamente el interés de los mismos tanto a corto plazo como a largo plazo.

Un primer campo de investigación podría ser la evaluación de los cambios en la demanda en países en los que se está valorando la posibilidad de implementar programas de GAD. Extrapolar las estimaciones de otros estudios previos probablemente no resultaría suficientemente preciso, por lo que sería conveniente realizar experiencias piloto que ayuden a cuantificar los patrones y magnitud de los cambios de la demanda.

Un segundo campo de investigación podría ser, dentro de la valoración del impacto de los cambios de la demanda en los sistemas eléctricos, la evaluación de dos aspectos escasamente explorados: los impactos en las redes y las interacciones con la generación intermitente. Por una parte, los impactos de la GAD en la operación y expansión de las redes apenas han sido investigados. Sería interesante estudiar, por ejemplo, los efectos en la calidad de servicio, o el potencial de las acciones de corto plazo para gestionar emergencias. Además de la complejidad técnica de estos análisis, debe tenerse en cuenta que los beneficios percibidos por compañías distribuidoras dependerán de forma crucial de la regulación vigente. Por otra parte, sería conveniente analizar cuidosamente la interacción de las medidas de GAD con la generación intermitente, para valorar el papel de la GAD como facilitadora de la integración de energías renovables. La dificultad de este análisis reside sobre todo en el carácter aleatorio tanto de la respuesta de la demanda como de la generación intermitente, y en el carácter secuencial de las acciones de GAD para compensar los desvíos de la generación.

Como conclusión positiva, la revisión del estado del arte revela que la evaluación de los beneficios de la gestión activa de la demanda es un campo de investigación muy activo y prometedor. En este artículo se han identificado y catalogado posibles líneas de investigación, lo cual puede resultar útil para orientar futuras investigaciones. 


\section{Referencias bibliográficas}

[1] AFFONSO, C. M.; DA SILVA, L. C. P. y FREITAS, W. (2006): Demand-side management to improve power security. Transmission and Distribution Conference and Exhibition, 2005/2006 IEEE PES, May.

[2] ALLCOTT, H. (2008): Real time pricing and imperfect competition in electricity markets. Working paper. Available from: http://economics.stanford.edu/seminars/real-timepricing-and-electricity-markets.

[3] ANDERSEN, F. M.; JENSEN, S. G.; LARSEN, H. V.; MEIBOM, P.; RAVN, H.; SKYTTE, K. y TOGEBY, M. (2006): Analyses of demand response in Denmark. Ris $\varnothing$ National Laboratory, Ris $\varnothing-\mathrm{R}-1565(\mathrm{EN})$, October.

[4] BAER, W.; FULTON, D. y MAHNOWSKI, S. (2004): Estimating the benefits of the GridWise initiative. Phase I report, May. Rand Science and Technology.

[5] BATLLE, C. y RODILLA, P. (2008): Electricity demand response tools: current status and outstanding issues. Working Paper IIT-08-006A. Prepared for: Special issue on incentives for a low-carbon energy future, European Review of Energy Markets.

[6] BERG, S. V.; CAPEHART, B. L.; FELDMAN, J. LATOUR, S. y SULLIVAN, R. L. (1983): An interdisciplinary approach to cost-benefit analysis of innovative electric rates. Resources and Energy, 5, pp. 313-330.

[7] BOONEKAMP, P. G. M. (2007): Price elasticities, policy measures and actual developments in household energy consumption - A bottom up analysis for the Netherlands. Energy Economics 29, pp. 133-157.

[8] BORENSTEIN, S. (2005): The long-run efficiency of real-time electricity pricing. The Energy Journal, 26, No. 3, pp. 93-116.

[9] BORENSTEIN, S.; JASKE, M. y ROSENFELD, A. (2002): Dynamic pricing, advanced metering and demand response in electricity markets. CSEM WP 105, University of California Energy Institute.

[10] BRAITHWAIT, S. D.; HANSEN, D. G. y KIRSCH, L. D. (2006): Incentives and rate designs for efficiency and demand response. Lawrence Berkeley National Laboratory. LBNL-60132.

[11] BRATTLE GROUP (2007): Quantifying demand response benefits in PJM. Prepared for PJM Interconnection, LLC and the Mid-Atlantic Distributed Resources Initiative (MADRI), January.

[12] CAPASSO, A.; GRATTIERI, W.; LAMEDICA, R. y PRUDENZI, A. (1994): A bottomup approach to residential load modeling. IEEE Transactions on Power Systems, 9, No. 2, May, pp. 957-964.

[13] CAPGEMINI (2007): Comparatif international des systèmes de télé-relève ou de télégestion et étude technico-économique visant à évaluer les conditions d'une migration du parc actuel de compteurs. March.

[14] CAVES, D. W.; CHRISTENSEN, L. R. y SCHOECH, P. E. (1984): A comparison of different methodologies in a case study of residential time-of-use electricity pricing Cost-benefit analysis. Journal of Econometrics, 26, pp. 17-34.

[15] CONCHADO, A. y LINARES, P. (2009a): Gestión activa de la demanda eléctrica: simulación de la respuesta de los consumidores domésticos a señales horarias de precio. IV Congreso AEEE, Sevilla. 
[16] CONCHADO, A. y LINARES, P. (2009b): Gestión activa de la demanda eléctrica doméstica: beneficios y costes. IIT Working Paper.

[17] CROSSLEY, D. (2008): Assessment and development of network-driven demand-side management measures. IEA Demand Side Management Programme, Task XV, Research Report No 2. NSW, Australia: Energy Futures Australia Pty Ltd.

[18] DARBY, S. (2006): The effectiveness of feedback on energy consumption: a review for DEFRA of the literature on metering, billing and direct displays. Environmental Change Institute, University of Oxford.

[19] DTE ENERGY (2007): Demand response overview and pilot concepts. July.

[20] EARLE, R.; KAHN, E. P. y MACAN, E. (2009): Measuring the capacity impacts of demand response. The Electricity Journal, 22, Issue 6, pp. 47-58.

[21] EC (2005): Green paper on energy efficiency or doing more with less. COM (2005) 265 final. Brussels: Commission of the European Communities.

[22] EEE (2006): A survey of Time-Of-Use (TOU) pricing and Demand-Response (DR) programs. Energy and Environmental Economics.

[23] EEI (2006): Responding to EPAct 2005: looking at smart meters for electricity, timebased rate structures, and net metering. Washington: Edison Electricity Institute.

[24] ERGEG (2007): Smart metering with a focus on electricity regulation. European Regulators' Group for Electricity and Gas. E07-RMF-04-03.

[25] ESC (2004): Mandatory rollout of interval meters for electricity consumers. Final decision, July. Essential Services Commission.

[26] FARUQUI, A. y GEORGE, S. S. (2002): The value of dynamic pricing in mass markets. The Electricity Journal, July, pp. 45-55.

[27] FARUQUI, A. y GEORGE, S. S. (2005): Quantifying customer response to dynamic pricing. The Electricity Journal, May, pp. 53-63.

[28] FARUQUI, A. y HLEDIK, R. (2007): The state of demand response in California. Prepared for California Energy Commission, final consultant report, CEC-200-2007-003F, September.

[29] FARUQUI, A. y SERGICI, S. (2009): Household response to dynamic pricing of electricity - A survey of the experimental evidence. Working paper series.

[30] FARUQUI, A.; HARRIS, D. y HLEDIK, R. (2009): Unlocking the $€ 53$ billion savings from smart meters in the EU. Discussion paper. The Brattle Group.

[31] FERC (2006): Assessment of demand response and advanced metering. Staff report, AD-06-2-000, Federal Energy Regulatory Commission.

[32] FRONTIER ECONOMICS (2006): Current prices, anybody? The costs and benefits of "smart" electricity meters.

[33] GOLDFINE, D.; HALDENSTEIN, M.; MONIZ, A. y TRAVERSO, D. (2008): Demand response review - A survey of major developments in demand response programs and initiatives. Washington: Edison Electric Institute.

[34] HANEY, A. B.; JAMASB, T. y POLLIT, M. (2009): Smart metering and electricity demand. Technology economics and international experience. Cambridge working paper in economics 0905, EPRG working paper 0903. Electricity Policy Research Group, University of Cambridge.

[35] HAUSMAN, J. A.; KINNUCA, M. y MCFADDEN, D. (1979): A two-level electricity demand model. Evaluation of the Connecticut time-of-day pricing test. Journal of Econometrics 10, pp. 263-289. 
[36] HEFFNER, G. (2007): A framework for demand response valuation. Demand Response Research Center.

[37] HERTER, K. (2007): Residential implementation of critical-peak pricing of electricity. Energy Policy 35, 2121-2130.

[38] HILL, L. J. (1991): Residential time-of-use pricing as a load management strategy. Effectiveness and applicability. Utilities Policy, July, 308-318.

[39] HOLLAND, S. P. y MANSUR, E. T. (2007): Is real-time pricing green? The environmental impacts of electricity demand variance. Working paper 1350 , October, National Bureau of Economic Research. Available from: http://www.nber.org/papers/w13508.

[40] IEA (2003). The power to choose - Demand response in liberalised electricity markets. ISBN: 92-64-10503-4. OECD/International Energy Agency.

[41] IEA (2008): World Energy Outlook. Paris: International Energy Agency.

[42] KÄRKKÄINEN, S. e IKÄHEIMO, J. (2009): Integration of demand side management with variable output DG. 10th IAEE European Conference, 7-10 September.

[43] KEMA-XENERGY (2003): Smart thermostat program impact evaluation. February.

[44] KING, C. S. y CHATTERJEE, S. (2003): Predicting California demand response. Public Utilities Fortnightly, July, pp. 27-32.

[45] KIRSCHEN, D. (2003): Demand-side view of electricity markets. IEEE Transactions on Power Systems, 18, May, No. 2, pp. 520-527.

[46] KOHLER, D. F. y MITCHELL, B.M. (1984): Response to residential time-of-use electricity rates - How transferable are the findings? Journal of Econometrics, 26, pp. 141177.

[47] LABANDEIRA, X. y LINARES, P. (2010): Energy efficiency: economics and policy. Journal of Economic Surveys. doi 10.1111/j.1467-6419.2009.00609.x

[48] LAWRENCE, A. y BRAITHWAIT, S. (1979): The residential demand for electricity with time-of-day pricing. Journal of Econometrics 9, pp. 59-77.

[49] LINARES, P. y CONCHADO, A. (2009): Gestión activa de la demanda eléctrica: Evaluación de su impacto en el sistema de generación. IV Congreso AEEE, Sevilla.

[50] LOCKHEED MARTIN ASPEN (2006): Demand response enabling technologies for small-medium businesses. Technical report prepared in conjunction with the $2005 \mathrm{Ca}$ lifornia Statewide Pricing Pilot. R.02.06.001.

[51] LU, N.; CHASSIN, D. P. y WIDERGREN, S. E. (2004): Simulating price responsive distributed resource. Power Systems Conference and Exposition, 2004 IEE PES.

[52] LUCAS, R. (1976): Econometric Policy Evaluation: A Critique, in Brunner, K.; Meltzer, A., The Phillips Curve and Labor Markets, Carnegie-Rochester Conference Series on Public Policy, 1, New York: American Elsevier, pp. 19-46.

[53] MEDLOCK III, K. B. (2004): The economics of energy demand. Encyclopedia of Energy. Elsevier Press, April.

[54] NAVIGANT CONSULTING (2005): Benefits of smart metering for Ontario. Discussion draft, April 5. Presented to Ontario Ministry of Energy.

[55] NEENAN, B. y HEMPHILL, R. (2008): Societal benefits of smart metering investments. The Electricity Journal, 21, October, Issue 8, pp. 32-45.

[56] NEENAN, B.; CAPPERS, P.; PRATT, D. y ANDERSON, J. (2005): Improving linkages between wholesale and retail markets through dynamic retail pricing. Prepared for New England ISO. Available at www.iso-ne.com. 
[57] NERA (2008): Cost-benefit analysis of smart metering and direct load control. Overview report for consultation. Report for the Ministerial Council on energy smart meter Working Group. NERA Economic Consulting.

[58] OFGEM (2006): Domestic Metering Innovation. February.

[59] PAATERO, J. V. y LUND, P. D. (2006): A model for generating household electricity load profiles. Preprint version. Available from: http://users.tkk.fi/patte/pub

[60] PARKS, R.W. y WEITZEL, D. (1984): Measuring the consumer welfare effects of timedifferentiated electricity prices. Journal of Econometrics, 26, pp. 35-64.

[61] PLMA (2002): Demand response: principles for regulatory guidance. Peak Load Management Alliance.

[62] REISS, P. C. y WHITE, M. W. (2005): Household electricity demand, revisited. Review of Economic Studies, 72, pp. 853-883.

[63] RRI (2008): The status of U.S. demand response. Research Reports International, 7th edition.

[64] RMI (2006): Demand response: an introduction - Overview of lessons, technologies, and lessons learned. Rocky Mountain Institute.

[65] SCE (2006): Inventory of emerging demand response technologies. Southern California Edison.

[66] SHAW, R.; ATTREE, M.; JACKSON, T. y KAY, M. (2009): The value of reducing distribution losses by domestic load-shifting: a network perspective. Energy Policy, 37, pp. 3159-3167.

[67] SIDERIUS, H. P. y DIJKSTRA, A. (2006): Smart metering for households: costs and benefits for the Netherlands. SenterNovem.

[68] SILVA, V. (2009): Value of smart appliances in systems balancing. Prepared for EIE project "Smart domestic appliances in sustainable energy systems" (SMART-A), WP 4.

[69] SIOSHANSI, R. y SHORT, W. (2009): Evaluating the impacts of real-time pricing on the usage of wind generation. IEEE Transaction on Power Systems, 24, No. 2, May, pp. 516-524.

[70] SPEES, K. y LAVE, L. B. (2007): Demand response and electricity market efficiency. The Electricity Journal 20, pp. 69-85.

[71] STANOJEVIĆ, V. y Silva, V. (2009): Role of demand side management to reduce system constraints and investment. Prepared for EIE project "Smart domestic appliances in sustainable energy systems" (SMART-A), WP 4.

[72] STANOJEVIĆ, V.; STRBAC, G.; SILVA, V.; LANG, P.; PUDJIANTO, D. y MACLEMAN, D. (2009): Application of storage and demand side management to optimise existing network capacity. CIRED, 20th International Conference on Electricity Distribution. Prague, 8-11 June.

[73] SU, C. y KIRSCHEN, D. (2009): Quantify the effect of demand response on electricity markets. IEEE Transactions on Power Systems, 24, August, No. 3, pp. 1199-1207.

[74] SUMMIT BLUE CONSULTING (2006): Evaluation of the 2005 Energy-Smart Pricing Plan. Summit Final report, January.

[75] US DOE (2006): Benefits of demand response in electricity markets and recommendations for achieving them. United States Department of Energy. February.

[76] VASCONCELOS, J. (2008): Survey of regulatory and technological developments concerning smart metering in the European Union electricity market. RSCAS Policy Papers 2008/01. Florence School of Regulation. 
[77] VIOLETTE, D.; FREEMAN, R. y NEIL, C. (2006a): Valuation and market analyses. Volume I: Overview. Prepared for: International Energy Agency, Demand Side Programme, January.

[78] VIOLETTE, D.; FREEMAN, R. y NEIL, C. (2006b): Valuation and market analyses. Volume II: Assessing the DRR benefits and costs. Prepared for: International Energy Agency, Demand Side Programme, January.

[79] WALAWALKAR, R.; BLUMSACK, S. APT, J. y FERNANDS, S. (2008): An economic welfare analysis of demand response in the PJM electricity market. Energy Policy 36, pp. 3692-3702.

[80] WEITZMAN, M. L. (1974): Prices versus quantities. Review of Economic Studies, 61(4), pp. 477-491.

[81] WOO, C. K. y HERTER, K. (2006): Residential demand response evaluation: a scoping study. LBNL-61090, collaborative report, Demand Response Research Center (DRCC), Ernest Orlando Lawrence Berkeley National Laboratory.

[82] ZIBELMAN, A. y KRAPELS, E. N. (2008): Deployment of demand response as a realtime resource in organized markets. The Electricity Journal 21, June, Issue 5, pp. 51-56. 